\title{
TERAPIA RACIONAL E INDUCCIÓN SUGESTIVA EN EL TRATAMIENTO DE CUADROS DEPRESIVOS
}

Responsable: Dr. Oscar Cáceres Moscoso Miembro: Blgo. Guillermo Bornaz Acosta

\section{RESUMEN}

Dentro del campo clínico de la psicologia, el presente estudio tiene como objetivo determinar la eficacia de una forma combinada de acción terapéutica de orden racional e inductivo en el tratamiento psicológico de cuadros depresivos, así como los tipos de depresión que pueden ser tratados con mayor éxito a través de esta forma de terapia mixta en función de la edad y sexo de los pacientes. Investigación realizada a través del trabajo de consultoría psicológica y el apoyo de la Facultad de Ciencias Médicas de la UNJBG de Tacna, con una población de 136 pacientes con diagnóstico de depresión nerviosa, atendidos durante el año 2005 y una muestra de 82 pacientes entre 17 y 63 años de edad, de los cuales 18 corresponden al sexo masculino y 64 al femenino, 28 son adolescentes y 54 adultos. Los resultados de la investigación nos hacen ver que la terapia racional, complementada con la inducción sugestiva, es apropiada y eficaz en el tratamiento de cuadros depresivos, tanto en pacientes jóvenes como adultos de ambos sexos por cuanto se profundiza mejor en el manejo y control terapéutico de los estados emocionales conflictivos, orientando y utilizando las propias capacidades cognitivas, creencias y estilos de vida del paciente y su entorno familiar.

Asimismo, la acción terapéutica de esta modalidad mixta hace más rápida la recuperación, tanto en cuadros depresivos leves como moderados y severos, en especial cuando no existe compromiso de daño orgánico, sin necesidad de acudir a fármacos, los mismos que sí cumplen una función complementaria en los casos de depresión profunda y especialmente asociados a otros cuadros patógenos.

\section{ABSTRACT}

Inside the clinic field of psychology this research has as a main objective determine the efficiency of a combine form of therapeutic action of rational order and induction in the treatment of depression cases, the types of depression can be treated with more success using this combine therapy in function to the age and sex of the patients. The research was made trough the psychological assessment and the support of the Medical Science Faculty in the National University of Tacna, with a population of 136 patients with de diagnosis of nervous depression, attended during the year 2005 our sample was 82 patients between 17 and 63 years old, 18 were male and 64 females,28 teenagers and 54 adults. The results of the research shows that the rational therapy complemented with suggestive induction, is appropriate in the treatment of depression in teenagers patient and adults of both sex, because the deep management of the patient and the therapeutic control of emotional conflicts, orienting and using the appropriate cognitive capacity, believes and style life of the patients and their families. Even though, the therapeutic action in this combine technique, make the recovery fast, so fast that the low and moderated and severe depression, specially when there is not organic damage, not using any drugs, that has a complementary function in deep depression associate to other pathogen cases.

\section{INTRODUCCIÓN}

En la actualidad, en nuestro medio, es alarmante la incidencia de pacientes que sufren cuadros depresivos y que acuden a hospitales y consultorios privados. Esta enfermedad, que afecta el estado emocional en general de la persona que la padece, mayormente ha sido tratada por médicos psiquiatras a través de fármacos inhibidores del sistema nervioso, algunas veces con buenos resultados y otras con serias limitaciones para su recuperación, aparte de las consecuencias colaterales que tienen el consumo alargado y muchas veces habitual que hacen fármaco-dependiente al depresivo. La depresión, como síndrome psicógeno, se caracteriza por el estado de permanente tensión, preocupación, angustia y desesperación, hoy en día es uno de los problemas psicológicos de mayor preocupación, por presentarse en los diversos estratos y edades poblacional es. Según datos estadísticos de la OMS, diez de cada cien mujeres y seis de cada cien hombres adultos sufren de algún tipo de depresión permanente. Hoy este cuadro se extiende peligrosamente a jóvenes adolescentes y niños de las diversas clases sociales, lo que explica la aparición cada vez mayor de suicidios en menores.

Las depresiones psicógenas se manifiestan en diversos grados, desde el nivel leve, moderado, severo a profundo, y en todas ellas las diversas formas de terapia psicológica se hacen muy largas y costosas, especialmente las que se basan en teorías psicoanalíticas que buscan superar causas individuales e inconcientes que han llevado al paciente a desarrollar el cuadro. Las teorías conductistas o neoconductistas, si bien presentan un tiempo más corto en el tratamiento, sus resultados, sea por condicionamiento clásico u operante, no siempre tienen la efectividad que se espera, especialmente si se dejan de ladolos 
factores socioafectivos que impactan enormemente en la aparición y desarrollo de cuadros depresivos. Quizá las terapias cognitivas tengan mejor en cuenta estos factores socioafectivos y cognoscitivos que suelen descuidar los psicólogos conductistas, pero dentro de ellas surge la inquietud de validar lo que muchos psicólogos hoy en día vienen proponiendo, el uso de terapias mixtas.

De alli que en el presente estudio formuláramos las siguientes interrogantes: ¿Cuál es la eficacia de la terapia racional e inducción sugestiva (como terapia mixta), en el tratamiento psicológico de cuadros depresivos?, ¿En qué tipo de depresión alcanza mayores éxitos la aplicación mixta de terapia psicológica racional e inducción sugestiva, teniendo en cuenta la edad y sexo de los pacientes?

No está demás señalar la enorme importancia que tiene el presente estudio, por la necesidad teórico-científica y aplicativa, de esclarecer en el campo de la psicología clínica y la salud pública, en general, el impacto de la aplicación de terapias mixtas en el tratamiento de los problemas psicológicos que enfrentan muchos pacientes en nuestra realidad social, como lo es la depresión nerviosa, que día a día va en aumento por las condiciones de vida que enfrentan, sean de orden económico, laboral, familiar, afectivo y otros, que indudablemente ejercen influencia poderosa en el estado socioafectivo y psicoemocional de la persona.

Por ello, como hipótesis de trabajo planteamos como enunciado que: el uso conjunto de terapia racional e inducción sugestiva ejerce una influencia favorable en el tratamiento de cuadros depresivos en pacientes jóvenes y adultos de distinto sexo. Hipótesis de trabajo sustentado en los siguientes principios teóricos de la ciencia psicológica actual:

\section{Terapia Racional:}

1. El pensamiento es el principal determinante de las emociones humanas, de allí la importancia de cómo interpretemos los acontecimientos y las cosas que pasan por nuestra mente.

2. El pensamiento disfuncional es la principal causa del malestar emocional. Por esto vemos en el paciente que lo que expresa o se dice a si mismo está provocando que se sienta de un modo $u$ otro $y$, lo que es peor, manteniendo su propia psicopatología.

3. Si la psicopatologia es producto del pensamiento irracional, lo mejor que podemos hacer es cambiar ese pensamiento. Pero cambiar ese pensamiento requiere de técnicas psicoterapéuticas apropiadas y no de fármacos.
4. Múltiples factores, tanto genéticos como las influencias ambientales, se encuentran en el origen del pensamiento irracional y la psicopatologia, por lo que parte de esa terapia psicológica debe ser reeducativa y reorientadora.

5. Si una persona evalúa su modo de pensar y lo cambia en el presente, su funcionamiento y sentimientos serán muy diferentes. Es decir, no es imprescindible (auque puede ayudar) ir al origen ni descubrir qué sucedió en el pasado, pues podemos trabajar directamente en el momento presente, estos nos diferencia de los psicoanalistas.

6. Las creencias irracionales se cambian mediante un esfuerzo activo y persistente para reconocerlas, enfrentarlas y modificarlas, lo cual constituye la tarea de la terapia racional psicológica

\section{Terapia Inductiva:}

1.Utilizar los recursos y talento de los pacientes, haciéndoles tomar conciencia, a través de la terapia racional inductiva, de sus capacidades y recursos que posee para su recuperación.

2.Visualización y afirmación positiva, para contrarrestar las afirmaciones y pensamientos negativos de personas con trastornos depresivos.

3. Reorientar los sentimientos y acciones alterados, haciendo ver al paciente que sus sentimientos, emociones y conductas pueden ser normales y adaptativos en el entorno en el que se desenvuelve.

4.Métodos de distracción, que incluye meditación, relajación y retroalimentación (feedback).

5.Resolución práctica de problemas, que incomodan o crean problemas emocionales al paciente.

6.Control del estado de ánimo y apoyo, conveniente para determinadas personas que se sienten incapaces e inseguras.

7.Potenciar la autoestima estimulando el desarrollo de aptitudes y confianza en aspectos positivos de vida.

\section{Análisis de la Información:}

Seguidamente, a manera de resumen, presentamos los cuadros estadisticos más relevantes de la investigación. 
CUADRO N ${ }^{\circ}$ 01: Niveles Depresivos en Hombres y Mujeres con Tratamiento Terapéutico Psicológico Racional Inductivo.

\begin{tabular}{|c|c|c|c|c|c|c|c|}
\hline \multirow{2}{*}{$\begin{array}{l}\text { Nivel } \\
\text { Depresivo }\end{array}$} & \multicolumn{2}{|c|}{ Hombres } & \multirow[t]{2}{*}{ Sexo } & \multicolumn{2}{|c|}{ Mujeres } & \multicolumn{2}{|c|}{ TOTAL } \\
\hline & $F$ & $\%$ & & $F$ & $\%$ & & \\
\hline a. leve & 6 & 7,3 & & 24 & 29,3 & 30 & 36,6 \\
\hline b. moderado & 8 & 9,8 & & 16 & 19,5 & 24 & 29,3 \\
\hline c. severo & 3 & 3,7 & & 19 & 23,2 & 22 & 26,8 \\
\hline d. Profundo & 1 & 1,2 & & 5 & 6,0 & 6 & 7,3 \\
\hline TOTAL & 18 & 22,0 & & 64 & 78,0 & 82 & 100,0 \\
\hline
\end{tabular}

Fuente: Historias clínicas psicológicas.

\section{Análisis Interpretativo del Cuadro $\mathrm{N}^{\circ} 01$}

En el Cuadro $\mathrm{N}^{\circ} 01$, con respecto a los niveles depresivos en hombres y mujeres con tratamiento terapéutico psicológico racional inductivo, observamos que, en mayor porcentaje, $78,0 \%$ son mujeres, de distintas edades. las que padecen cuadros depresivos, de los cuales en mayor proporción son de tipo leve $29,3 \%$ y moderado $19,5 \%$. En cambio el $22,0 \%$ restante de depresiones corresponde a hombres de distintas edades de los cuales el mayor porcentaje es de tipo moderado $9,8 \%$. Esto reafirma el criterio general de que son las mujeres las que en su mayoría enfrentan problemas de orden depresivo, debido a factores de mayor receptividad que caracterizan a la mujer y las condiciones socioculturales dificiles que enfrentan en una sociedad aún machista: quehaceres del hogar junto al trabajo, cuidado de los hijos y otros.

CUADRO N 02: Nivel de Depresión y Tiempo de Tratamiento Racional Inductivo.

\begin{tabular}{|l|r|r|r|r|r|r|r|r|}
\hline \multirow{3}{*}{ Depresión } & \multicolumn{3}{|c|}{ Tiempo de tratamiento } & \multicolumn{5}{|c|}{ TOTAL } \\
\cline { 2 - 8 } & 1 a 2 meses & 2 a 4 meses & \multicolumn{4}{|c|}{ 4 a 6 meses } \\
\cline { 2 - 9 } & $\mathbf{F}$ & $\%$ & \multicolumn{1}{|c|}{ F } & $\%$ & F & $\%$ & F & $\%$ \\
\hline a. Leve & 28 & 34,2 & 2 & 2,4 & & - & 30 & 36,6 \\
\hline b. moderado & 14 & 17,1 & 10 & 12,2 & & - & 24 & 29,3 \\
\hline c. severo & 6 & 7,3 & 8 & 9,7 & 8 & 9,8 & 22 & 26,8 \\
\hline d. Profundo & 1 & 1,2 & 3 & 3,7 & 2 & 2,4 & 6 & 7,3 \\
\hline TOTAL & 49 & 59,8 & 23 & 28,0 & 10 & 12,2 & 82 & 100,0 \\
\hline
\end{tabular}

Fuente: Historias clinicas psicológicas.

\section{Análisis Interpretativo del Cuadro $\mathrm{N}^{\circ} 02$}

En el Cuadro $\mathrm{N}^{\circ} 02$, referente al nivel de depresión y tiempo de tratamiento racional inductivo, observamos que, en un alto porcentaje, $59,8 \%$ de pacientes sometidos a este tratamiento mixto superan la depresión en un tiempo reducido de 1 a 2 meses, en especial cuando se trata de depresiones leves y moderadas, puesto que las severas y profundas demoran entre 2 y 6 meses. Aun así, estos resultados reafirman nuestra hipótesis en el sentido de que la práctica terapéutica psicológica mixta racional inductiva, es uno de los tratamientos de gran efectividad, en el campo de la psicología, que permite tratar los cuadros depresivos con efectividad y en tiempos cortos.

CUADRO No 03: Niveles Depresivos y Tratamiento Terapéutico Psicológico Racional Inductivo con o sin Apoyo de Fármacos.

\begin{tabular}{|c|c|c|c|c|c|c|}
\hline \multirow{3}{*}{$\begin{array}{c}\text { Nivel } \\
\text { Depresivo }\end{array}$} & \multicolumn{4}{|c|}{ Terapia racional inductiva } & \multirow{2}{*}{\multicolumn{2}{|c|}{ TOTAL }} \\
\hline & \multicolumn{2}{|c|}{ Con fármacos } & \multicolumn{2}{|c|}{ Sin fármacos } & & \\
\hline & $\mathbf{F}$ & $\%$ & $\mathbf{F}$ & $\%$ & $\mathbf{F}$ & $\%$ \\
\hline a. Leve & - & - & 30 & 36,6 & 30 & 36,6 \\
\hline b. moderado & - & - & 24 & 29,3 & 24 & 29,3 \\
\hline c. severo & 5 & 6,1 & 17 & 20,7 & 22 & 26,8 \\
\hline d. Profundo & 5 & 6,1 & 1 & 1,2 & 6 & 7,3 \\
\hline TOTAL & 10 & 12,2 & 72 & 87,8 & 82 & 100,0 \\
\hline
\end{tabular}

Fuente: Historias clínicas psicológicas.

\section{Análisis Interpretativo del Cuadro $\mathrm{N}^{\circ} 03$}

En el Cuadro $\mathrm{N}^{\circ} 03$, con respecto a los niveles depresivos y el tratamiento terapéutico psicológico racional inductivo con o sin el apoyo de fármacos prescritos por el médico, observamos que, en alto porcentaje, $87,8 \%$ de pacientes no necesitaron el apoyo de fármacos, en especial cuando se trata de depresiones leves y moderadas. En el caso de depresiones severas, de 22 pacientes solo 5 necesitaron el apoyo de fármacos y 17 no las necesitaron. Sin embargo, en las depresiones profundas, de 6 pacientes 5 si necesitaron fármacos.

Estos resultados nos hacen ver que la terapia psicológica tiene efectos extraordinarios en el tratamiento de depresiones leves y moderadas y aun severas sin necesidad de fármacos; y solo cuando se trata de depresiones profundas, que mayormente están asociadas a otro tipo de alteraciones físicas o mentales, sí es necesario recurrir al apoyo farmacológico de un médico, en cuyo caso se recomienda el tratamiento interdisciplinario médico y psicológico.

CUADRO N 04: Hombres y Mujeres que se sintieron Recuperados de la Depresión, Luego del Tratamiento Terapéutico Psicológico Racional Inductivo.

\begin{tabular}{|c|c|c|c|c|c|c|}
\hline \multirow{3}{*}{$\begin{array}{c}\text { Superación } \\
\text { de la } \\
\text { depresión }\end{array}$} & \multicolumn{4}{|c|}{ Sexo } & \multirow{2}{*}{\multicolumn{2}{|c|}{ TOTAL }} \\
\hline & \multicolumn{2}{|c|}{ Hombres } & \multicolumn{2}{|c|}{ Mujeres } & & \\
\hline & $F$ & $\%$ & $F$ & $\%$ & $F$ & $\%$ \\
\hline a. Totalmente & 16 & 19,6 & 59 & 71,9 & 75 & 91,5 \\
\hline b. Medianamente & 2 & 2,4 & 5 & 6,1 & 7 & 8,5 \\
\hline c. Ninguna & -- & - & - & & & \\
\hline TOTAL & 18 & 22,0 & 64 & 78,0 & 82 & 100,0 \\
\hline
\end{tabular}

Fuente: Historias clínicas psicológicas.

\section{Análisis Interpretativo del Cuadro $\mathrm{N}^{\circ} 04$}

En el Cuadro $\mathrm{N}^{\circ}$ 04, correspondiente a los hombres y mujeres que se sintieron recuperados de la depresión, luego del tratamiento terapéutico psicológico racional inductivo, observamos que en mayor porcentaje, tanto en hombres $19,6 \%$, como en 
mujeres 71,9\%; afirmaron que se sintieron totalmente recuperados de la depresión, es decir, el 91,5\% de pacientes que conforman la muestra de estudio se sintieron totalmente recuperados y sólo el $8,5 \%$ restante se sintieron medianamente recuperados; pero lo importante es que ningún paciente se sintió con ninguna recuperación. El $100 \%$ de pacientes sintieron recuperación de la depresión, lo que reafirma nuestra hipótesis de lo positivo y favorable de la acción terapéutica psicológica racional inductiva.

CUADRO N 05 : Adolescentes y Adultos con Depresión que Participaron de la Terapia Psicológica Racional Inductiva.

\begin{tabular}{|l|r|r|r|r|r|r|}
\hline \multirow{2}{*}{$\begin{array}{l}\text { Nivel } \\
\text { Depresivo }\end{array}$} & \multicolumn{4}{|c|}{ Sexo } & \multicolumn{2}{|c|}{ T O TA L } \\
\cline { 2 - 7 } & \multicolumn{2}{|c|}{ Hombres } & \multicolumn{2}{c|}{ Mujeres } & \multicolumn{1}{c|}{ O T } \\
\cline { 2 - 7 } & F & \multicolumn{1}{c|}{$\%$} & F & \multicolumn{1}{c|}{$\%$} & \multicolumn{1}{c|}{ F } & $\%$ \\
\hline a. Leve & 18 & 21,9 & 12 & 14,6 & 30 & 36,6 \\
\hline b. moderado & 6 & 7,3 & 18 & 22,0 & 24 & 29,3 \\
\hline c. severo & 4 & 4,9 & 18 & 22,0 & 22 & 26,8 \\
\hline d. Profundo & - & & 6 & 7,3 & 6 & 7,3 \\
\hline TOTAL & 28 & 34,1 & 54 & 65,9 & 82 & 100,0 \\
\hline
\end{tabular}

Fuente: Historias clínicas psicológicas.

\section{Análisis Interpretativo del Cuadro $\mathrm{N}^{\circ} 05$}

En el Cuadro $N^{\circ} 05$, referido a los adolescentes y adultos con depresión que participaron de la terapia psicológica racional inductiva, observamos que, en mayor porcentaje, $65,9 \%$ son adultos, pero lo significativo es que hay un buen porcentaje de $34,1 \%$ que son adolescentes los que acudieron a terapia psicológica racional inductiva por depresión. Por otro lado, se observa que en porcentajes más elevados está apareciendo la depresión leve $36,6 \%$ y la moderada $29,3 \%$ en ambos grupos. Sin embargo, los adolescentes presentan una tendencia mayor a sufrir depresiones leves, mientras que los adultos presentan más depresiones moderadas y severas, pero que en todos los casos la terapia psicológica racional inductiva tiene efectos favorables en su recuperación.

\section{CONCLUSIONES}

1. La terapia racional complementada con la inducción sugestiva, como tratamiento psicológico, es apropiada y eficaz en el tratamiento de cuadros depresivos, tanto en pacientes jóvenes como adultos de ambos sexos.
2. La terapia racional emotiva debe ser complementada con las técnicas de inducción sugestiva, a través de la implementación y desarrollo cuidadoso de un plan terapéutico, para cada paciente y de manera única, por el psicólogo en el campo clínico.

3. Las técnicas terapéuticas de tratamiento psicológico en el campo de la psicología clínica, y dentro de ellas las de tipo racional inductivas, exigen del profesional psicólogoe una preparación especializada pero a su vez una gran capacidad de análisis y creatividad para plasmar programas terapéuticos eficaces en el tratamiento de la depresión.

4. Los cuadros depresivos, hoy en día, vienen apareciendo en mayor incidencia, tanto en adultos como en jóvenes adolescentes, mayormente ocasionados por los problemas difíciles de la vida que les toca enfrentar, por lo cual es necesario realizar acciones preventivas, tanto a nivel psicológico como médico y educativo.

5. El tipo de depresión donde alcanzan mayores éxitos la combinación de terapias racionales e inductivas es en las llamadas depresiones leves y moderadas, tanto en jóvenes como en adultos de ambos sexos.

6. La terapia racional inductiva puede tener éxitos apropiados en los pacientes para superar la depresión de tipo severa y profunda, siempre y cuando no exista compromiso o daño orgánico, en cuyo caso es necesario el complemento médico especializado, para un tratamiento interdisciplinario.

\section{BIBLIOGRAFÍA}

Coleman Y OTROS (1990): Psicología de la anormalidad Ed. Trillas, México.

Garfield SOL L. (1990): Psicología clínica.

Ed. Manual Moderno, México.

Goleman, DANIEL (1997):La salud emocional.

Ed. Kairós, España.

MC Kay Y OTROS (1986): Técnicas cognitivas para el tratamiento del estrés y depresión.

Ed. Martinez Roca, España.

Kerlinger, FRED (1998): Investigación del comportamiento.

Ed. Mc Graw-Hill, México.

Sandler Y Davidson (1980): Psicopatología.

Ed. Trillas, México. 